\title{
63-jährige Patientin mit progredienter Gangstörung, Blickrichtungsnystagmus und kalten Händen
}

\author{
Autoren \\ Jan Heckelmann, Maike Dohrn, Manuel Dafotakis \\ Institut \\ Neurologische Klinik, Universitätsklinik der RWTH Aachen \\ Bibliografie \\ DOI https://doi.org/10.1055/a-1015-1011 \\ Klin Neurophysiol 2019; 50: 239-240 \\ (c) Georg Thieme Verlag KG Stuttgart · New York \\ ISSN 1434-0275
}

\author{
Korrespondenzadresse \\ Priv.-Doz. Dr. M. Dafotakis \\ Neurologische Klinik \\ Universitätsklinik der RWTH Aachen \\ Pauwelsstraße 30 \\ 52074 Aachen \\ mdafotakis@ukaachen.de
}

Die Vorstellung der 63-jährigen Patientin erfolgte aufgrund einer seit ca. zwei Jahren progredienten Gangstörung und Schwindel. Zudem berichtete sie über sehr „kalte Hände“. Kognitiv habe sie keine Einschränkungen bemerkt, was der begleitende Ehemann bestätigte. Auf Nachfragen gab die Patientin noch an, dass sie in den letzten Monaten vermehrt Schwierigkeiten habe, rechtzeitig die Toilette zu erreichen, so dass es schon einige Male „schief gegangen" sei.

Klinisch fanden sich an auffälligen Befunden ein Blickrichtungsnystagmus horizontal und ein Kopfschüttelnystagmus. Die VORSupression war gestört bei normalem Halmagyi-Kopfimpulstest. Das Stehen war schwankend ohne relevante Veränderung bei Augenschluss, das Gehen ataktisch unsicher bei normalem Muskeltonus. Finger-Nase- und Knie-Hacke-Versuch waren beidseits gestört. Die Muskeleigenreflexe waren stgl. mittellebhaft auslösbar, und es ergaben sich keine Pyramidenbahnzeichen. Die Sensibilität war seitengleich, jedoch waren die Hände - wie von der Patientin bereits erwähnt - eiskalt, dabei aber eher dunkelviolett verfärbt. Im Rahmen der differenzialdiagnostischen Erwägungen wurde eine cMRT angefertigt ( $\triangleright$ Abb. 1).

\section{DIE CMRT AUFNAHME ZEIGT EINEN TYPISCHEN BEFUND ( $\triangleright$ ABB. 1) \\ ? Wie lautet dieser? \\ ? Welche (Verdachts-)Diagnose können Sie anhand der Klinik und der Bildgebung stellen? \\ ? Passen die kalten Hände zu Ihrer Verdachtsdiagnose und welchen weiteren klinischen Test würden Sie noch vorschlagen, um die Diagnose zu erhärten?}

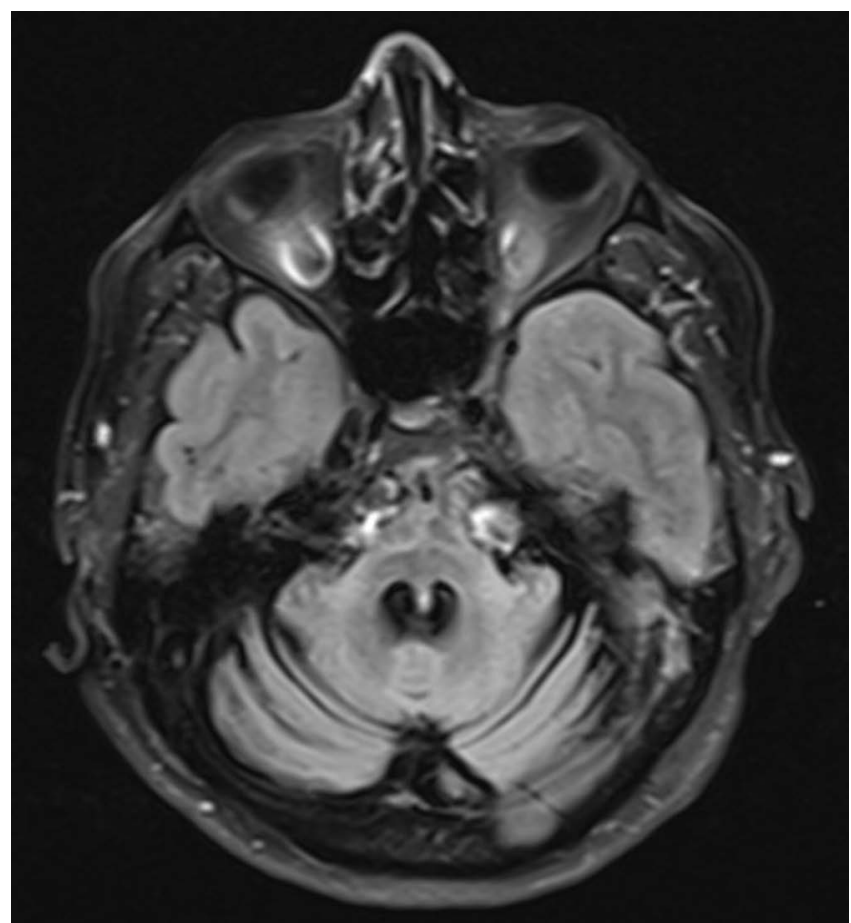

- Abb. 1 axiale Schnittführung einer cMRT in FLAIR-Wichtung. 


\section{Auflösung}

\section{cMRT Befund}

In der cMRT findet sich ein so genanntes „hot cross bun“- oder auch „Kreuzungszeichen“, welches als pathognomonisch für eine Multisystematrophie anzusehen ist. Die Bezeichnung „hot cross bun“ rührt daher, dass bei der englischen Variante der Osterbrötchen ein Kreuz eingekerbt wird, welches ähnlich dem Befund in der cMRT ist. Darüber hinaus kann in unserem Fall auch eine Pons- und Kleinhirnatrophie diagnostiziert werden ( $\triangleright$ Abb. 2).

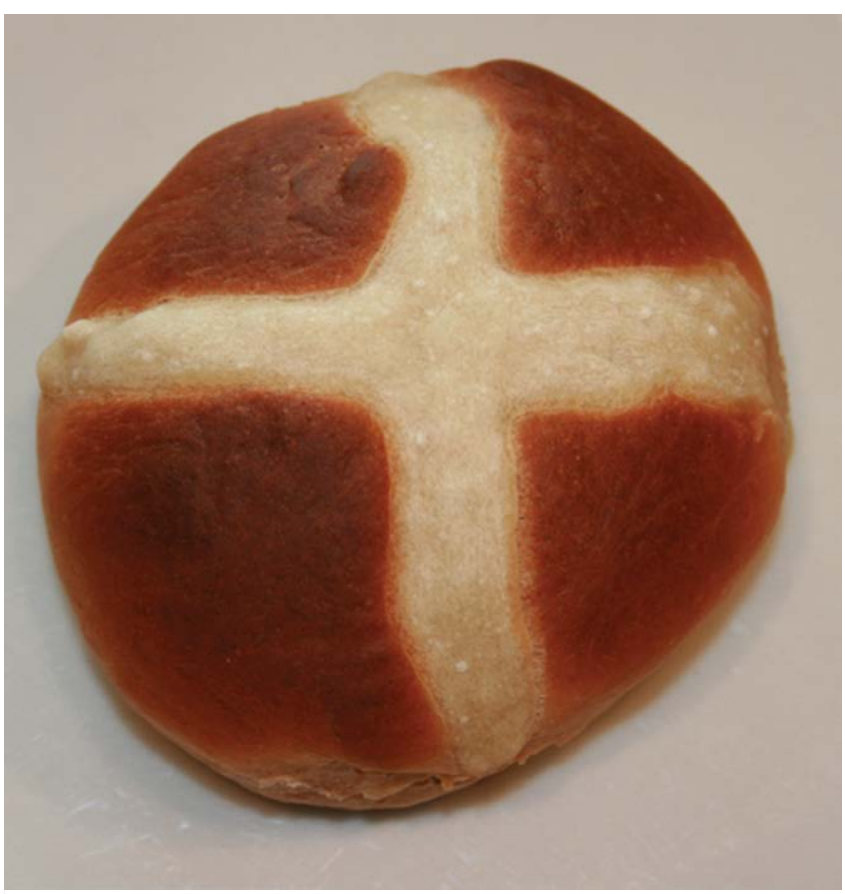

- Abb. 2 „Hot cruss bun“, Osterbrötchen aus Großbritannien.

\section{Verdachtsdiagnose}

Anamnese, Klinik und Verlauf sowie die Bildgebung erlauben die Diagnose einer

\section{Multisystematrophie vom cerebellären Typ (MSAc)}

\section{Kalte Hände und weitere klinische Tests}

An weiteren klinischen Tests ist in dieser Konstellation ein Schellong-Test (der eine schwere orthostatische Hypotonie zeigt) sinnvoll. Aufgrund der gestörten vasomotorischen Kopplung kommt es bei einer Vielzahl der Patienten mit MSA auch zu kalten Extremitäten, was diagnostisch genutzt werden kann [1].

\section{Interessenkonflikt}

Die Autoren geben an, dass kein Interessenkonflikt besteht.

\section{Literatur}

[1] Klein C, Brown R, Wenning G et al. The "cold hands sign" in multiple system atrophy. Mov Disord 1997; 12: 514-518 